\title{
Relationship between Autonomic Nervous System Activity during Sleep and Fasting Glucose in Japanese Workers
}

\author{
Naoki KAMATA ${ }^{1 *}$, Katsutoshi TANAKA ${ }^{2}$, Shiho MORITA ${ }^{3}$, Hirokuni TAGAYA ${ }^{4}$, \\ Masatoshi KAWASHIMA ${ }^{5}$, Masayoshi SHICHIRI ${ }^{6}$ and Hitoshi MIYAOKA ${ }^{7}$
}

${ }^{1}$ Department of Psychiatry, Graduate School of Medical Sciences, Kitasato University, 1-15-1 Kitasato, Minami-ku, Sagamihara, Kanagawa 252-0374, Japan

${ }^{2}$ Department of Occupational Mental Health, Graduate School of Medical Sciences, Kitasato University, 1-15-1 Kitasato, Minami-ku, Sagamihara, Kanagawa 252-0374, Japan

${ }^{3}$ Hygiene Management Center, Kakogawa Works, Kobe Steel, Ltd., 1 Kanazawa-cho, Kakogawa, Hyogo 675-0185, Japan

${ }^{4}$ Department of Health Science, School of Allied Health Sciences, Kitasato University, 1-15-1 Kitasato, Minami-ku, Sagamihara, Kanagawa 252-0374, Japan

${ }^{5}$ Department of Preventive Medicine and Public Health, Graduate School of Medical Sciences, Kitasato University Graduate School, 1-15-1 Kitasato, Sagamihara, Minami-ku, Kanagawa 252-0374, Japan

${ }^{6}$ Department of Endocrinology, Diabetes and Metabolism, School of Medicine, Kitasato University, 1-15-1 Kitasato, Sagamihara, Minami-ku, Kanagawa 252-0374, Japan

${ }^{7}$ Department of Psychiatry, School of Medicine, Kitasato University, 1-15-1 Kitasato, Minami-ku, Sagamihara, Kanagawa 252-0374, Japan

Received November 26, 2010 and accepted January 5, 2011

Published online in J-STAGE June 21, 2011

\begin{abstract}
Although autonomic nervous system activity is reportedly related to diurnal glucose tolerance impairment, the relationship with glucose tolerance during sleep is unclear. Since work styles have recently diversified, it is important to assess the effect of sleep on workers' health. Elucidation of the relationship between autonomic nervous system activity during sleep and glucose tolerance in workers may facilitate preventive measures against diabetes using nonpharmacological means (e.g., sleep hygiene education, relaxation techniques and stress management). We examined whether autonomic nervous system activity during sleep is related to fasting glucose or glycated hemoglobin (HbA1c) in individuals with either normal or impaired fasting glucose tolerance. The subjects were 77 apparently healthy Japanese workers with normal or impaired fasting glucose. We used high frequency (HF) and the ratio of low frequency to high frequency (LF/HF) obtained by pulse wave analysis to estimate autonomic nervous system activity. The data were analyzed using a generalized estimating equation adjusted for potential confounders (age, gender, engagement in shift work, sleep duration, and body mass index). Fasting glucose was significantly negatively related to HF, the parasympathetic component during sleep. Our results suggest that parasympathetic activity during sleep is associated with fasting glucose in apparently healthy Japanese workers.
\end{abstract}

Key words: Autonomic nervous system activity, Heart rate variability, Fasting glucose, HbAlc, Frequency domain

*To whom correspondence should be addressed.

E-mail: naokikamat@gmail.com 


\section{Introduction}

Autonomic nervous system activity can be assessed relatively easily with low invasiveness by analyzing heart rate variability. Analysis of autonomic nervous system activity based on heart rate variability uses timedomain and frequency-domain analyses. Time-domain analysis calculates the standard deviation of all normalto-normal heart beat intervals over a 24 -h period and the root mean square of successive differences of adjacent normal-to-normal intervals, while frequency-domain analysis calculates high frequency (HF) and the ratio of low frequency to high frequency (LF/HF ratio) $)^{1}$.

Previous studies have attempted to elucidate whether these autonomic nervous indices are involved in the development and progression of a number of diseases ${ }^{2-6)}$. In diabetes, these indices are used to assess dysautono$\mathrm{mia}^{2}$. It has been suggested that autonomic nervous system activity levels are involved during the daytime in both diabetes and impaired glucose tolerance. The Atherosclerosis Risk In Communities Study carried out in the United States followed 8,185 individuals for approximately $8 \mathrm{yr}$ and demonstrated that the prevalence of type 2 diabetes mellitus was significantly higher in those with a higher heart rate or decreased heart rate variability observed during daytime 2-min electrocardiogram (ECG) recordings ${ }^{7}$ ). The Framingham Heart Study investigated 1,919 subjects who had heart rate variability measured for $2 \mathrm{~h}$ during the day using a portable electrocardiograph, and it demonstrated that fasting glucose levels were significantly correlated with LF, HF, and the LF/HF ratio ${ }^{8}$. A Taiwanese study using 1,440 subjects employing frequency-domain analysis of daytime 5-min ECG recordings revealed that HF was significantly lower and the LF/HF ratio was higher in the impaired glucose tolerance and diabetes mellitus groups than in the normal glucose group ${ }^{9}$.

Accumulating lines of evidence indicate a relationship between glucose tolerance and sleep. A study conducted using 1,486 subjects demonstrated that sleep duration was associated with an increased prevalence of impaired glucose tolerance and diabetes ${ }^{10)}$. Tasali et al. ${ }^{11)}$ demonstrated that suppression of slow wave sleep without any change in total sleep duration causes a decrease in glucose tolerance and insulin sensitivity, while a reduction in sleep duration might increase sympathetic nerve activity $^{12)}$. Furthermore, parasympathetic nerve activity is higher during slow wave sleep than in other sleep phases ${ }^{13)}$. Therefore, it is generally believed that autonomic nervous system activity is involved in the relationship between sleep and glucose tolerance; however, this relationship has not yet been clarified.

In recent years, sleep hygiene education and coun- termeasures against metabolic syndrome have been initiated in some companies. In the field of occupational health, previous studies have also examined glucose tolerance in relation to autonomic nervous system activity. One study of the effect of night shift work on autonomic nervous system activity showed that the longer the duration of night shift work, the smaller the HF and LF components become. The results of that study might explain the positive association between the duration of night shift work and the risk of cardiovascular disease $^{14)}$. In a study involving 223 Japanese workers, a long commute time and long overtime hours were found to reduce parasympathetic components ${ }^{15)}$.

Since work styles have recently diversified, it is important to assess the effect of sleep on the health of workers. Elucidation of the relationship between autonomic nervous system activity during sleep and glucose tolerance in workers may facilitate preventive measures against diabetes using non-pharmacological means (e.g., sleep hygiene education, relaxation techniques and stress management). The present study was designed to examine the relationship between autonomic nervous system activity during sleep and fasting glucose or glycated hemoglobin (HbAlc) by a wearable sensor. We obtained HF and LF/HF ratio data by pulse wave analysis recorded during the entire sleep period for 3 consecutive nights in apparently healthy workers with normal fasting glucose and those with impaired fasting glucose tolerance.

\section{Subjects and Methods}

\section{Participants}

Seventy-seven apparently healthy Japanese workers (64 men and 13 women) who received annual medical checkups sponsored by their employers in the Japanese manufacturing industry were included as subjects after obtaining written informed consent. These workers were from three work sites: steel, beverage and petrochemical manufacturers, each employing approximately 300 to 500 workers. Of 77 subjects, 8 workers (10.4\%), 7 males and 1 female, worked in three 8-h shifts. The day shift was 08:00-16:00, the evening shift was 16:00 $-00: 00$ and the night shift was 00:00-08:00. They worked under a 1-wk rotating shift schedule, which usually comprised a cycle of 5 consecutive work days followed by $2 \mathrm{~d}$ off. Working shifts were the same in all three sites. The inclusion criteria specified that workers should be informed of the study's purpose and be given a consent form during medical checkups conducted by each company. Patients who were diagnosed as having any diseases, including those currently under medical treatment, were excluded from the study. 
In addition, patients with a fasting blood glucose of $126 \mathrm{mg} / \mathrm{dl}$ or more and who were classified as diabetes type by the Japan Diabetes Association classification ${ }^{16)}$, were excluded, because they were most likely to have diabetic autonomic neuropathy. The present study was conducted during regular medical checkups in the spring of 2008 and 2009, with blood test and pulse wave measurements performed at the same time. A schedule was planned so that shift workers would receive blood test and pulse wave measurements during the daytime of the working week. The details of the study were approved by the ethics committee of Kitasato University.

\section{Biochemical analysis}

The subjects received blood tests in the morning after at least a 12-h fast. For fasting blood glucose and $\mathrm{HbA1c}$, the results of blood tests at regular medical checkups were used. However, HbAlc was measured only in subjects for whom it was considered necessary based on fasting blood glucose values taken during regular checkups in the previous year or in those subjects requested by industrial physicians to take the test. Fasting glucose was classified based on the criteria issued by the Japan Diabetes Association in 2010 in a report by its committee on the diagnostic criteria of diabetes mellitus: normal fasting glucose is $<110 \mathrm{mg} / \mathrm{dl}$ (normal type) and impaired fasting glucose is $110-125 \mathrm{mg} / \mathrm{dl}$ (borderline type) ${ }^{16)}$. HbAlc was expressed as recommended by the Japan Diabetes Society as follows. The value for HbA1c (\%) was estimated as a National Glycohemoglobin Standardization Program (NGSP) equivalent value (\%) calculated by the formula $\mathrm{HbA1c}(\%)=\mathrm{HbA1c}$ (Japan Diabetes Society value) $(\%)+0.4 \%{ }^{16)}$.

\section{Measurement and analysis of heart rate variability and sleep duration}

A wearable physiological sensor (NEM-T1, Toshiba, Japan) with a built-in pulse wave sensor and actigraph was used to measure heart rate variability and sleep duration for 3 consecutive nights. For taking measurements, the subjects were asked to attach the sensor to themselves and to turn it on when they went to bed and to turn off the sensor and detach it from themselves when they got up. The data on pulse-peak intervals (PPIs) and the amount of activity stored in the sensor were then transferred to a PC via a USB connection for subsequent analysis using a dedicated analysis software package (NEM-SS1, Toshiba, Japan). The sensor was $50 \times 60 \times 13 \mathrm{~mm}$ in size and $35 \mathrm{~g}$ in weight, and came with a rechargeable battery. A 3-axis accelerometer and a 4 GB memory were built in to the sensor. It was also accompanied by an outside sensor (pulse wave sensor).
The sensor was attached to the wrist with an accompanying wristband like a watch and the pulse wave sensor was attached to a finger. The sampling rate of the pulse wave and 3-axis accelerations was $64 \mathrm{~Hz}$. However, the resolution of the PPIs was $0.1 \mathrm{~ms}$ using linear interpolation to detect the pulse peak. This sensor detects body motion by an acceleration of $0.01 \mathrm{G}$ or higher, which is the same as an actigraph ${ }^{17,18)}$.

Autonomic nervous system activity indices were computed using a software package (NEM-SS1, Toshiba, Japan) accompanying the sensor, from the frequency analysis of PPI variations. First, the PPI dataset sampled in 1 min was interpolated at even intervals by cubic spline interpolation by the min. Fast Fourier transform was then executed for the even-interval PPIs to obtain the frequency spectrum. In the frequency domain, the integral value of the power from $0.04 \mathrm{~Hz}$ to $0.15 \mathrm{~Hz}$ is called LF, which shows both sympathetic and parasympathetic nervous activities. The integral value of the power from $0.15 \mathrm{~Hz}$ to $0.4 \mathrm{~Hz}$ is called HF, which shows parasympathetic nervous activity ${ }^{17)}$. The ratio of these elements is employed to express sympathetic nerve activity with the LF/HF ratio ${ }^{1)}$. The analysis software automatically calculates the sleep duration based on the algorithm of the Cole equation from the body motion detected by the actigraph ${ }^{18)}$.

The validity of the sensor is explained as follows. First, the correlation coefficient between the amount of activity counted by the sensor worn on the left forearm and that measured by an actigraph (MicrominiMotionlogger Actigraph, Ambulatory Monitoring Inc.) worn on the right forearm during sleep was 0.95 . The correlation coefficients between the PPIs computed by the pulse wave measured by the sensor and the R-R intervals computed by a simultaneously measured ECG during sleep were also evaluated. Single-channel ECG was measured by the CM5 lead using polysomnography (Polymate AP1124, TEAC Corporation, sampling rate: $1 \mathrm{kHz}$ ) simultaneously with the PPIs measured by this sensor. R-R intervals were computed using commercially available R-R interval analysis software for polysomnography (NoruPro Light Systems, Japan). The correlation coefficient was $0.96^{17}$ ).

\section{Statistical analysis}

We first determined Pearson's correlations of fasting glucose and $\mathrm{HbA} 1 \mathrm{c}$ with the $\mathrm{HF}$ and $\mathrm{LF} / \mathrm{HF}$ ratios. In univariate analysis, the mean values of data obtained in 3 nights were used for the $\mathrm{HF}$ and $\mathrm{LH} / \mathrm{HF}$ ratios. The autonomic nervous system components, which were not normally distributed, were subjected to logarithmic transformation to calculate the $\log \mathrm{HF}$ and $\log \mathrm{LF} / \mathrm{HF}$ ratio. To perform analysis after adjusting for potential 
Table 1. Attributes of the participants

\begin{tabular}{llllr}
\hline & Total $(\mathrm{n}=77)$ & Males $(\mathrm{n}=64)$ & Females $(\mathrm{n}=13)$ & $p$ value $^{1)}$ \\
\hline Age $(\mathrm{yr})$ & $41.0(10.7)$ & $41.8(10.4)$ & $36.9(11.6)$ & 0.19 \\
BMI $\left(\mathrm{kg} / \mathrm{m}^{2}\right)$ & $23.8(4.0)$ & $23.8(3.5)$ & $24.0(5.8)$ & 0.36 \\
Fasting blood sugar level (mg/dl) & $91.0(10.0)$ & $92.2(9.4)$ & $84.8(11.0)$ & $<0.01$ \\
HF (ms) & $31.86(20.62)$ & $28.90(19.2)$ & $35.12(12.77)$ & 0.35 \\
LF/HF ratio & $1.35(0.55)$ & $1.41(0.46)$ & $0.98(0.36)$ & $<0.01$ \\
Sleep duration (min) & $346.5(91.8)$ & $347.8(95.7)$ & $340.2(72.5)$ & 0.17 \\
Engagement in shift work, $\mathrm{n}(\%)$ & $8(100)$ & $7(87.5)$ & $1(12.5)$ & $<0.01$ \\
HbAlc $(\%)[\mathrm{n}]$ & $5.6(0.3)[\mathrm{n}=19]$ & $5.6(0.3)[\mathrm{n}=18]$ & $5.1(-)[\mathrm{n}=1]$ & $<0.01$ \\
\hline
\end{tabular}

Metric variables are shown as the mean (SD). Categorical variables are shown as $\mathrm{n}(\%)$. Sleep duration was detected by a sensor.

Abbreviations: BMI: body mass index; HF: high frequency component; LF: low frequency component; HbA1c: glycated hemoglobin.

1) To test for differences between sexes for each variable, continuous variables were tested by $t$-test and categorical variables were tested by Fisher's exact test.

confounders, i.e., age, gender, body mass index (BMI), engagement in shift work, and sleep duration, a generalized estimating equation (GEE) model was used, with fasting glucose levels and $\mathrm{HbA1c}$ as dependent variables. In the GEE, records containing missing values can be used in analysis. If measurements are performed multiple times in the same individual, it is possible to perform analysis without reducing statistical power by fixing an appropriate working correlation matrix and introducing individuals as a random effect ${ }^{19,20)}$. In the analyses, HF, LH/HF ratio, age, gender, BMI, engagement in shift work, and sleep duration, were introduced into the model as a fixed effect, as well as individuals as a random effect. HF and $\mathrm{LH} / \mathrm{HF}$ ratios did not show a normal distribution. These were divided into tertiles and introduced into the model as continuous variables. We constructed a linear regression model using an exchangeable working correlation matrix. The data were analyzed using the PASW version 17.0 software program (SPSS Japan Inc, Japan). A value of $p<0.05$ was used to denote a statistically significant difference.

\section{Results}

Attributes of the participants are shown in Table 1. Seventy-three subjects $(94.8 \%)$ were found to have normal fasting glucose $(<110 \mathrm{mg} / \mathrm{dl})$ and 4 subjects $(5.2 \%)$ had impaired fasting glucose (110-125 mg/dl). Only 19 subjects underwent $\mathrm{HbA1c}$ measurement.

Correlation coefficients of autonomic nervous system components with fasting glucose and $\mathrm{HbAlc}$ are shown in Table 2. Pearson's correlation coefficients were significant between fasting glucose and log HF $(p<0.01)$ and the $\log \mathrm{LF} / \mathrm{HF}$ ratio $(p=0.03)$. Pearson's correlation coefficient between $\mathrm{HbAlc}$ and $\log \mathrm{HF}$ was significant $(p=0.02)$. Pearson's correlation coefficient between
Table 2. Correlation coefficients of autonomic nervous system components with fasting glucose $(n=77)$ and HbA1c $(n=19)$

\begin{tabular}{lrr}
\hline & Correlation coefficient & $p$ value \\
\hline Fasting glucose - log HF & -0.308 & $<0.01$ \\
Fasting glucose - log LF/HF ratio & 0.254 & 0.03 \\
HbA1c - log HF & -0.546 & 0.02 \\
HbA1c - log LF/HF ratio & 0.299 & 0.21 \\
\hline
\end{tabular}

logHF: HF after logarithmic transformation; log LF/HF: LF/HF ratio after logarithmic transformation.

Table 3. The relationship between fasting glucose and autonomic nervous system activity during sleep

\begin{tabular}{lllr}
\hline HRV indices & $\mathrm{B}^{1)}$ & $(95 \% \mathrm{CI})$ & $p$ value \\
\hline $\mathrm{HF}^{2)}$ & -1.579 & $(-2.461 \sim-0.697)$ & $<0.001$ \\
$\mathrm{LF}^{2} \mathrm{HF}$ ratio & \\
& -0.380 & $(-1.462 \sim 0.701)$ & 0.49
\end{tabular}

1) Partial regression coefficient adjusted for age, gender, BMI, shift work, and sleep duration.

2) $\mathrm{HF}$ and $\mathrm{LH} / \mathrm{HF}$ ratios were divided into tertiles and introduced into the model as continuous variables.

Abbreviations: HRV: heart rate variability; B: partial regression coefficient; CI: confidence interval; BMI: body mass index; HF: high frequency component; LF: low frequency component.

$\mathrm{HbA} 1 \mathrm{c}$ and the $\log \mathrm{LF} / \mathrm{HF}$ ratio was not significant $(p=0.21)$.

Table 3 shows the results of analysis using a GEE of the relationships of fasting glucose adjusted for potential confounders. Fasting glucose was significantly negatively related to HF $(p<0.001)$. There was no significant relationship between fasting glucose and the LF/HF ratio.

Table 4 shows the relationship between $\mathrm{HbAlc}$ and autonomic nervous system activity during sleep, analyzed by the same method as mentioned above. There was no significant relationship between $\mathrm{HbA1c}$ and both autonomic nervous system components. 
Table 4. The relationship between HbA1c and autonomic nervous system activity during sleep

\begin{tabular}{lllr}
\hline HRV indices & $\mathrm{B}^{1)}$ & $(95 \% \mathrm{CI})$ & $p$ value \\
\hline $\mathrm{HF}^{2)}$ & -0.047 & $(-0.105 \sim 0.011)$ & 0.113 \\
$\mathrm{LF} / \mathrm{HF} \mathrm{ratio}^{2)}$ & -0.013 & $(-0.073 \sim 0.046)$ & 0.66 \\
\hline
\end{tabular}

1) Partial regression coefficient adjusted for age, gender, BMI, shift work, and sleep duration.

2) $\mathrm{HF}$ and $\mathrm{LH} / \mathrm{HF}$ ratios were divided into tertiles and introduced into the model as continuous variables.

Abbreviations: HRV: heart rate variability; B: partial regression coefficient; CI: confidence interval; BMI: body mass index; HF: high frequency component; LF: low frequency component.

\section{Discussion}

The results of our study identified that a significant negative correlation was present between HF and fasting glucose during sleep. Since there were only 4 subjects in the impaired fasting glucose group, we did not divide the population into a normal fasting glucose group and impaired fasting glucose group for statistical analysis. With regard to the difference in HbAlc results between univariate analysis and multivariate analysis, the consistency of results appeared to be reduced because $\mathrm{HbAlc}$ was determined in only a limited amount of subjects with a resulting decrease in statistical power.

Previous studies on the relationship between sleep and glucose tolerance used the subjective component of sleep quality along with sleeping time to assess sleep ${ }^{21,22)}$. The only previous study in which objective physiological indices were used employed an electroencephalograph to examine slow wave sleep, glucose tolerance and insulin resistance ${ }^{11)}$. In addition, only one study, by Perciaccante et al. ${ }^{23)}$, has examined the relationship between glucose tolerance or insulin resistance and autonomic nervous system activity during nighttime. They examined autonomic nervous system activity over a 1-d period, the relationship between LF, the sympathetic component at night (from 00:00 h to 06:00 h), and the homeostasis model assessment-index, which is an index of insulin resistance, and reported that the higher the LF at night, the greater the insulin resistance. Thus, they focused on the circadian rhythm of the autonomic nervous system and demonstrated a relationship between sympathetic nerve activity at night and insulin resistance ${ }^{23)}$.

Although a number of previous studies have examined heart rate variability in diabetic patients ${ }^{24-29)}$, only a few studies have examined the relationship of normal fasting glucose and impaired fasting glucose with heart rate variability $8,23,30,31$. We have shown, for the first time, that there is a relationship between autonomic nervous system activity during sleep and fasting glucose in apparently healthy workers with either normal fasting glucose or with impaired fasting glucose.

The measurement of cardiovascular autonomic nervous system activity can be performed simply and with low-invasiveness ${ }^{1)}$; generally, an electrocardiograph is used to take measurements and brief recordings in the resting position are made in the examination room. However, to obtain long recordings, it is necessary to have a portable electrocardiograph attached and to also take into account constraints in terms of measurement locations and attachment to relatively large equipment. However, the advantage of our study is that it was possible to assess actual autonomic nervous system activity during sleep at home by using a small sensor that hardly disrupts sleep. Although autonomic nervous system activity during the day is affected by various daily activities $^{32,33)}$, since measurements were taken during sleep in this study, we believed that these effects were minimal and that measurements were taken in a relatively stable manner.

The results of our study suggest that parasympathetic stimulation during sleep at night may improve glucose tolerance. For parasympathetic nervous system during sleep at night to be predominant, relaxation techniques (e.g., breathing techniques, progressive muscle relaxation, appropriate music, and aromatherapy) can be applied, as well as techniques to reduce stimulation to the sympathetic nervous system (e.g., coping with stress, limiting the intake of caffeine and other stimulants, and adjusting the lighting) $)^{34)}$. Studies need to be conducted to verify whether sleep guidance may be effective for causing parasympathetic nervous system during sleep to be predominant, thereby improving impaired glucose tolerance and insulin resistance.

A limitation of this study is that no adjustments were made for any confounders that may have affected autonomic nervous system activity, e.g., smoking, caffeine intake, exercise habits, and diet. In this study, an oral glucose tolerance test was not performed. Therefore, it was impossible to completely exclude patients with diabetes mellitus. In addition, diabetes is one of the most common causes of autonomic neuropathy $9,35,36$ ), and cardiac autonomic dysfunction is a complication that can be easily overlooked ${ }^{37)}$. In our study, we measured normal fasting glucose and impaired fasting glucose based on the supposition that diabetic autonomic neuropathy would not affect the results of this study; however, this was not possible to verify. In our study, there was a limit to the compatibility of units for the data on the autonomic nervous system. In general, LF and HF are expressed as the area $\left(\mathrm{ms}^{2}\right)$ obtained by fast Fourier transform analysis, but this equipment measures the peak height (ms) of every component in 
the areas obtained to approximately express LF and HF. Autonomic nervous system activity is affected by body position $^{33)}$, but in our study, measurements were taken without controlling for any shift in body position during sleep or for any differences in posture between individuals.

Suppression of slow wave sleep by sleep fragmentation has been reported to significantly reduce glucose effectiveness and insulin sensitivity ${ }^{38)}$. In the present study, because sleep evaluation by an electroencephalograph was not performed, it was not possible to assess the effect of sleep stages on glucose tolerance. Although there are limitations to our study, our results suggest that parasympathetic activity during sleep is associated with fasting glucose in apparently healthy Japanese workers.

\section{Acknowledgements}

The present study was supported by a Grant-in-Aid (No. 20590613) from the Japan Society for the Promotion of Science.

\section{References}

1) Task Force of the European Society of Cardiology and the North American Society of Pacing and Electrophysiology (1996) Heart rate variability: standards of measurement, physiological interpretation and clinical use. Circulation 93, 1043-65.

2) Vinik AI, Maser RE, Mitchell BD, Freeman R (2003) Diabetic autonomic neuropathy. Diabetes Care 26, 1553-79.

3) de Sá JC, Costa EC, da Silva E, Zuttin RS, da Silva EP, Lemos TM, De Azevedo GD (2011) Analysis of heart rate variability in polycystic ovary syndrome. Gynecol Endocrinol 27, 443-7.

4) Spiegelhalder K, Fuchs L, Ladwig J, Kyle SD, Nissen C, Voderholzer U, Feige B, Riemann D (2011) Heart rate and heart rate variability in subjectively reported insomnia. J Sleep Res 20, 137-45.

5) van Zyl LT, Hasegawa T, Nagata K (2008) Effects of antidepressant treatment on heart rate variability in major depression: a quantitative review. Biopsychosoc Med 2, 12.

6) Bonnemeier H, Hartmann F, Wiegand UK, Irmer C, Kurz T, Tölg R, Katus HA, Richardt G (2000) Heart rate variability in patients with acute myocardial infarction undergoing primary coronary angioplasty. Am J Cardiol 85, 815-20.

7) Carnethon MR, Golden SH, Folsom AR, Haskell W, Liao D (2003) Prospective investigation of autonomic nervous system function and the development of type 2 diabetes: the Atherosclerosis Risk In Communities study, 1987-1998. Circulation 107, 2190-5.
8) Singh JP, Larson MG, O'Donnell CJ, Wilson PF, Tsuji H, Lloyd-Jones DM, Levy D (2000) Association of hyperglycemia with reduced heart rate variability (The Framingham Heart Study). Am J Cardiol 86, 309-12.

9) Wu JS, Yang YC, Lin TS, Huang YH, Chen JJ, Lu $\mathrm{FH}$, Wu CH, Chang CJ (2007) Epidemiological evidence of altered cardiac autonomic function in subjects with impaired glucose tolerance but not isolated impaired fasting glucose. J Clin Endocrinol Metab 92, 3885-9.

10) Gottlieb DJ, Punjabi NM, Newman AB, Resnick HE, Redline S, Baldwin CM, Nieto FJ (2005) Association of sleep time with diabetes mellitus and impaired glucose tolerance. Arch Intern Med 165, 863-7.

11) Tasali E, Leproult R, Ehrmann DA, Van Cauter E (2008) Slow-wave sleep and the risk of type 2 diabetes in humans. Proc Natl Acad Sci U S A 105, 1044-9.

12) Knutson KL, Van Cauter E (2008) Associations between sleep loss and increased risk of obesity and diabetes. Ann NY Acad Sci 1129, 287-304.

13) Trinder J, Kleiman J, Carrington $M$, Smith S, Breen S, Tan N, Kim Y (2001) Autonomic activity during human sleep as a function of time and sleep stage. J Sleep Res 10, 253-64.

14) Togo F, Takahashi M (2009) Heart rate variability in occupational health - a systematic review. Ind Health 47, 589-602.

15) Kageyama T, Nishikido N, Kobayashi T, Kurokawa Y, Kabuto M (1997) Commuting, overtime, and cardiac autonomic activity in Tokyo. Lancet 350, 639.

16) Seino $Y$, Nanjo K, Tajima N, Kadowaki T, Kashiwagi A, Araki A, Ito T, Inagaki N, Iwamoto Y, Kasuga M, Hanafusa T, Haneda M, Ueki K (2010) The committee of Japan Diabetes Society on the diagnostic criteria of diabetes mellitus. Report of the committee on the classification and diagnostic criteria of diabetes mellitus. Tonyobyo (Diabetes) 53, 450-67 (in Japanese).

17) Suzuki T, Ouchi K, Kameyama K, Takahashi M (2009) Development of a sleep monitoring system with wearable vital sensor for home use. BIODEVICES, 326-31, Published by BIOSTEC.

18) Cole RJ, Kripke DF, Gruen W, Mullaney DJ, Gillin JC (1992) Automatic sleep/wake identification from wrist activity. Sleep 15, 461-9.

19) Diggle PJ, Heagerty P, Liang K-Y, Zeger SL (2002) Analysis of Longitudinal Data (2nd Ed.), 284, Oxford University Press, Oxford.

20) Twisk JWR (2003) Applied Longitudinal data Analysis for Epidemiology: a practice gude. 208-12, Cambridge University Press, Cambridge.

21) Punjabi NM (2009) Do sleep disorders and associated treatments impact glucose metabolism? Drugs 69 (Suppl 2), 13-27.

22) Tasali E, Leproult R, Spiegel K (2009) Reduced sleep duration or quality: relationships with insulin resistance and type 2 diabetes. Prog Cardiovasc Dis 51, 381-91. 
23) Perciaccante A, Fiorentini A, Paris A, Serra P, Tubani L (2006) Circadian rhythm of the autonomic nervous system in insulin resistant subjects with normoglycemia, impaired fasting glycemia, impaired glucose tolerance, type 2 diabetes mellitus. BMC Cardiovasc Disord 6, 19.

24) Tsuji H, Venditti FJ Jr, Manders ES, Evans JC, Larson MG, Feldman CL, Levy D (1996) Determinants of heart rate variability. J Am Coll Cardiol 28, 1539-46.

25) Wawryk AM, Bates DJ, Couper JJ (1997) Power spectral analysis of heart rate variability in children and adolescents with IDDM. Diabetes Care 20, 1416-21.

26) Frattola A, Parati G, Gamba P, Paleari F, Mauri G, Di Rienzo M, Castiglioni P, Mancia G (1997) Time and frequency domain estimates of spontaneous baroreflex sensitivity provide early detection of autonomic dysfunction in diabetes mellitus. Diabetologia 40, 1470-5.

27) Rutter MK, McComb JM, Brady S, Marshall SM (1998) Autonomic neuropathy in asymptomatic subjects with non-insulin-dependent diabetes mellitus and microalbuminuria. Clin Auton Res 8, 251-7.

28) Thomaseth K, Cobelli C, Bellavere F, Balzani I, De Masi G, Bax G, Carenza P (1990) Heart rate spectral analysis for assessing autonomic regulation in diabetic patients. J Auton Nerv Syst 30 (Suppl), S169-71.

29) Liao D, Cai J, Brancati FL, Folsom A, Barnes RW, Tyroler HA, Heiss G (1995) Association of vagal tone with serum insulin, glucose, and diabetes mellitusThe ARIC Study. Diabetes Res Clin Pract 30, 211-21.

30) Stein PK, Barzilay JI, Domitrovich PP, Chaves PM, Gottdiener JS, Heckbert SR, Kronmal RA (2007) The relationship of heart rate and heart rate variability to non-diabetic fasting glucose levels and the metabolic syndrome: the Cardiovascular Health Study. Diabet Med 24, 855-63.

31) Schönauer M, Thomas A, Morbach S, Niebauer J, Schönauer U, Thiele H (2008) Cardiac autonomic diabetic neuropathy. Diab Vasc Dis Res 5, 336-44.

32) Fraguas R Jr, Marci C, Fava M, Iosifescu DV, Bankier B, Loh R, Dougherty DD (2007) Autonomic reactivity to induced emotion as potential predictor of response to antidepressant treatment. Psychiatry Res 151, 169-72.

33) Cohen H, Matar MA, Kaplan Z, Kotler M (1999) Power spectral analysis of heart rate variability in psychiatry. Psychother Psychosom 68, 59-66.

34) Kakinuma M, Takahashi M, Kato N, Aratake $Y$, Watanabe M, Ishikawa Y, Kojima R, Shibaoka M, Tanaka K (2010) Effect of brief sleep hygiene education for workers of an information technology company. Ind Health 48, 758-65.

35) Spallone V, Menzinger G (1997) Diagnosis of cardiovascular autonomic neuropathy in diabetes. Diabetes 46 (Suppl 2), S67-S76.

36) Gerritsen J, Dekker JM, TenVoorde BJ, Kostense PJ, Heine RJ, Bouter LM, Heethaar RM, Stehouwer CD (2001) Impaired autonomic function is associated with increased mortality, especially in subjects with diabetes, hypertension, or a history of cardiovascular disease: the Hoorn Study. Diabetes Care 24, 1793-8.

37) Maser RE, Lenhard JM, DeCherney SG (2000) Cardiovascular autonomic neuropathy: the clinical significance of its determination. Endocrinologist 10, 27-33.

38) Stamatakis KA, Punjabi NM (2010) Effects of sleep fragmentation on glucose metabolism in normal subjects. Chest 137, 95-101. 